\title{
GLAM: Glycogen-derived Lactate Absorption Map for visual analysis of dense and sparse surface reconstructions of rodent brain structures on desktop systems and virtual environments
}

\author{
Marco Agus $^{\mathrm{a}, *}$, Daniya Boges ${ }^{\mathrm{b}}$, Nicolas Gagnon ${ }^{\mathrm{b}}$, Pierre J. Magistretti ${ }^{\mathrm{b}}$, Markus Hadwiger ${ }^{\mathrm{a}}$, \\ Corrado Calí ${ }^{b}$ \\ a Visual Computing Center, King Abdullah University of Science and Technology, Thuwal 23955-6900, Saudi Arabia \\ ${ }^{\mathrm{b}}$ Biological and Environmental Science and Engineering Division, King Abdullah University of Science and Technology, Thuwal 23955-6900, Saudi Arabia
}

\section{A R T I C L E I N F O}

\section{Article history:}

Received 4 December 2017

Revised 3 April 2018

Accepted 29 April 2018

Available online $\mathrm{xxx}$

\section{Keywords:}

Scientific visualization

Neuroenergetics

3D interactive visual analysis

Nanometric scale brain reconstructions

Virtual Reality in Neuroscience

\begin{abstract}
A B S T R A C T
Human brain accounts for about one hundred billion neurons, but they cannot work properly without ultrastructural and metabolic support. For this reason, mammalian brains host another type of cells called "glial cells", whose role is to maintain proper conditions for efficient neuronal function. One type of glial cell, astrocytes, are involved in particular in the metabolic support of neurons, by feeding them with lactate, one byproduct of glucose metabolism that they can take up from blood vessels, and store it under another form, glycogen granules. These energy-storage molecules, whose morphology resembles to spheres with a diameter ranging $10-80$ nanometers roughly, can be easily recognized using electron microscopy, the only technique whose resolution is high enough to resolve them. Understanding and quantifying their distribution is of particular relevance for neuroscientists, in order to understand where and when neurons use energy under this form. To answer this question, we developed a visualization technique, dubbed GLAM (Glycogen-derived Lactate Absorption Map), and customized for the analysis of the interaction of astrocytic glycogen on surrounding neurites in order to formulate hypotheses on the energy absorption mechanisms. The method integrates high-resolution surface reconstruction of neurites, astrocytes, and the energy sources in form of glycogen granules from different automated serial electron microscopy methods, like focused ion beam scanning electron microscopy (FIB-SEM) or serial block face electron microscopy (SBEM), together with an absorption map computed as a radiance transfer mechanism. The resulting visual representation provides an immediate and comprehensible illustration of the areas in which the probability of lactate shuttling is higher. The computed dataset can be then explored and quantified in a 3D space, either using 3D modeling software or virtual reality environments. Domain scientists have evaluated the technique by either using the computed maps for formulating functional hypotheses or for planning sparse reconstructions to avoid excessive occlusion. Furthermore, we conducted a pioneering user study showing that immersive VR setups can ease the investigation of the areas of interest and the analysis of the absorption patterns in the cellular structures.
\end{abstract}

(c) 2018 Elsevier Ltd. All rights reserved.

\section{Introduction}

Neurons are the most popular cell type of mammalian brains, accounting for its fundamental function, learning and memory. Their capacity to form plastic connections, the synapses, to interface with each other and form networks that allows for processing and storing information and performing tasks based on environmental inputs, makes the understanding of their physiology at all

\footnotetext{
* Corresponding author.

E-mail addresses: marco.agus@kaust.edu.sa (M. Agus), corrado.cali@kaust.edu.sa (C. Calí).
}

spatial and temporal scale of the outmost importance. Nevertheless, neurons cannot possibly sustain such humongous set of tasks, from action potential to synaptic transmission, without proper cellular and molecular support. In order to sustain neurons to do their difficult job, another, less popular but equally important class of cells is present in the brain, called glial cells, nowadays referred to by experts as "the other half of the brain". Under the umbrella of glial cells falls a number of cell types whose phylogeny is very diverse, although all of them are devoted to maintain the homeostasis in the central and peripheral nervous system. For instance, microglial cells have mesoderal origin, represent the innate immunitary response system of the brain; they are profoundly different 
from oligodendrocytes, whose role is to produce myelin sheets for axons, in order to increase of several fold the speed of propagation of action potentials [1]. The energetic support of neurons, among other functions, is sustained by another class of glial cells, called astrocytes. This function is well known since over a century, as it was first hypothesized by Camillo Golgi amid observation that the majority of these cells possess a so called "vascular endfoot", which he though was likely to be the mechanism of redistribution of nutrients from the vasculature to the brain parenchyma [2]. Neuroenergetics is indeed to date one of the top rated fields related to the investigation of the astrocytic involvement in brain physiological functioning [3]. Working on the energy support of the brain has been shown to be beneficial under several pathological conditions, such as stroke [4] and epilepsy [5] to mention a few. Although mammalian cells produce energy under the form of ATP from glucose, recent evidence suggest that under intense activity, neurons are preferentially using another molecule, an intermediate product of glucose metabolism, the (in)famous lactate acid (or simply lactate) as fuel for their cellular respiration cycle to produce energy. Neurons cannot produce lactate themselves, and when they need it they rely on astrocytes that are then able to shuttle to them. This mechanism is called astrocyte-neuron lactate shuttle (ANLS: [6]). But, because the lactate support from astrocytes to neurons is not continuous, astrocytes can uptake and store glucose, to keep an energy reserve ready for its usage when necessary. Such storage mechanism is called glycogen, a branched polymer of glucose easily recognizable under electron microscopy as a dense, round molecule, with a diameter ranging 20-80 nanometers roughly [7-9]. In the brain, astrocytes specifically express glycogen granules, and possess the enzymes that allows for its synthesis from glucose, as well as their breakdown to lactate; it is therefore legit to consider glycogen as an energy storage mechanism, being the precursor of lactate, the energy substrate that astrocytes shuttle to neurons. In a recent study, researchers took advantage of a high-resolution imaging technique, based on blockface scanning electron microscopy (SEM), to be able to also clearly recognize astrocytic processes, as well as the most greedy consumers of energy, synapses. In order to quantify the spatial distribution of glycogen in three dimensions, the electron microscope was coupled to a module allowing for automated serial sectioning and imaging modules, the focused ion beam (FIB-SEM) [10], to acquire high resolution image stacks whose segmentation results in accurate three-dimensional models of brain structures that eventually were analyzed with the aid of virtual reality setups [11]. High-throughput imaging techniques used in neuroscience are all based on automated serial sectioning, like the aforementioned FIB-SEM, but also others like the serial block face module 3 View (SBFEM), all falling under the generic term of 3DEM. The consequent use of the generated $3 \mathrm{D}$ models is pushing neuroscientists, as a community, to put efforts in the creation of hybrid methods for investigating aspects of brain physiology [12]. In the last five years, a number of papers were published that demonstrate how customized existing tools, like Blender [11,13-15], or custommade softwares [16-19] are a valuable approach for using 3D models for producing high level scientific papers. Inspired by previous work on the quantification of glycogen distribution in a reconstruction from rat hippocampus [11] we present here an illustrative visualization method suited for the interactive visual analysis of the influence of glycogen on surrounding neurites, namely axons and dendrites, as well as their synaptic elements, boutons and spines, respectively, in the interest of understanding the shuttling mechanisms of lactate [20]. The method takes as input a high-resolution surface reconstruction of neural structure and a list of energy sources in the form of glycogen granules, and computes an influence map according to a radiance transfer mechanism (see Fig. 1). By considering a photon mapping analogy, areas of greater glycogen concentration will highlight portions of the cellular plasma membranes where the glycogen-derived lactate shuttling is more likely to occur. Compared to the previous study [11], which quantified the contribution of glycogen limited to the preor post-synaptic terminal metabolism, based on a criteria of nearest neighbor, this approach is more unbiased because the photon mapping does not exclude the potential effect a single granule on more than one target, and is evaluated on the entire neuropil to highlight even structures other than synapses, like dendritic shafts. This framework has been tested by neuroscientists for formulating neuroenergetics hypotheses about the utilization of glycogen derived energy. Moreover, as full reconstructions of every structures on an imaged volume (called "dense reconstruction") after results in an overly cluttered scene, which is hard to interpret, we suggest here alternative use as a tool to either filter the view to only structures showing an appreciable absorption peak, or to pre-select structures to undergo segmentation and subsequent 3D reconstruction. We here extend our contribution to Smart Tool and Applications in Graphics [21], by putting particular efforts in the integration of the absorption model and the glycogen analysis framework with a pioneering interactive exploration on virtual environment employing head mounted displays. This framework was tested on dense reconstructions from rat hippocampus, somatosensory cortex from adult and aged mice where the effect of aging could be tested, and on a dataset involving learning mice, where only the astrocyte and the glycogen were reconstructed, to use the GLAM map for planning a sparse reconstruction. We discuss also the outcomes of a user evaluation that compared the desktop application and the VR application, involving 27 subjects, with expertise in the field ranging from medium to high, which is the first reported in literature to our knowledge. Results show how the integration of the GLAM model inside Virtual Reality system improves the perception of absorption patterns and speed up the exploration of the cellular structures, demonstrating the usefulness of this tool for neuroanatomy.

\section{Related work}

\subsection{Rationale}

More and more, computer graphics is making its way through neurobiology, and the field that is pushing this as well as other technologies (such as imaging and image processing and segmentation, etc...) to their forefront is connectomics. Such impressive technological advancement is beneficial for many other fields of neuroscience; for instance, recently the intracellular endoplasmatic reticulum and mitochondria from cortical neurons have been extensively reconstructed and analyzed in 3D [22]. These advancements allowed to upscale imaging and consequently reconstructions to larger volumes; as a result, scientists needed to come up with strategies to simplify such huge datasets, to benefit correlative analysis and interpretation. One example is the Neurolines tool [19], that allows easily to read complex connectivity maps extracted from densely reconstructed neuronal networks as a 2D subway map.

\subsection{Neuroscience domain: astrocytes and glycogen metabolism role}

It is generally acknowledged how astrocytes play a number of pivotal roles in the support of neuronal functioning throughout one's life-time. Astrocytes are involved in a variety of processes in the brain including fine tuning of synaptic transmission [23], neuroprotection and neurometabolism [3], however the underlining mechanism for these processes is far from being fully understood. Moreover, the recent discovery of the astrocyte neuron lactate shuttle (ANLS) has proved quintessential among astrocytes various 

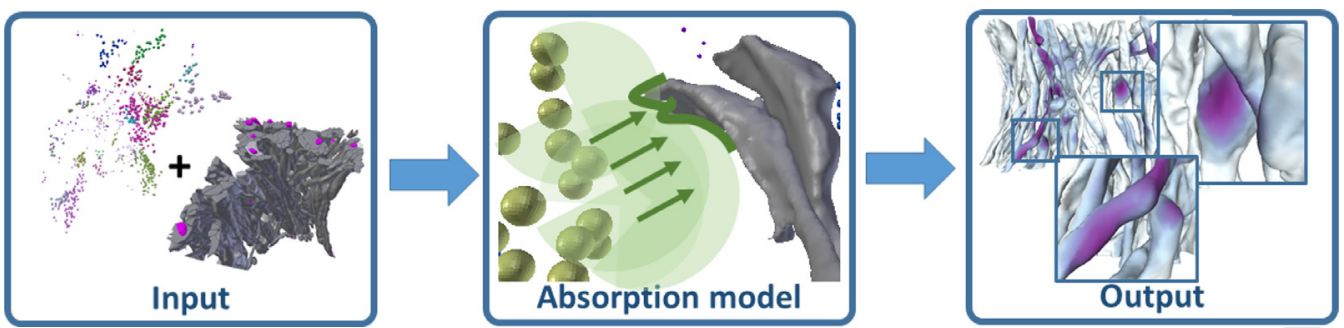

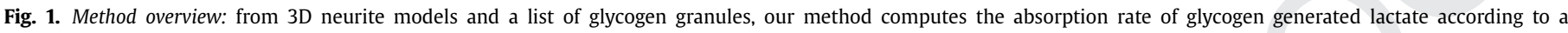
radiance transfer mechanism.
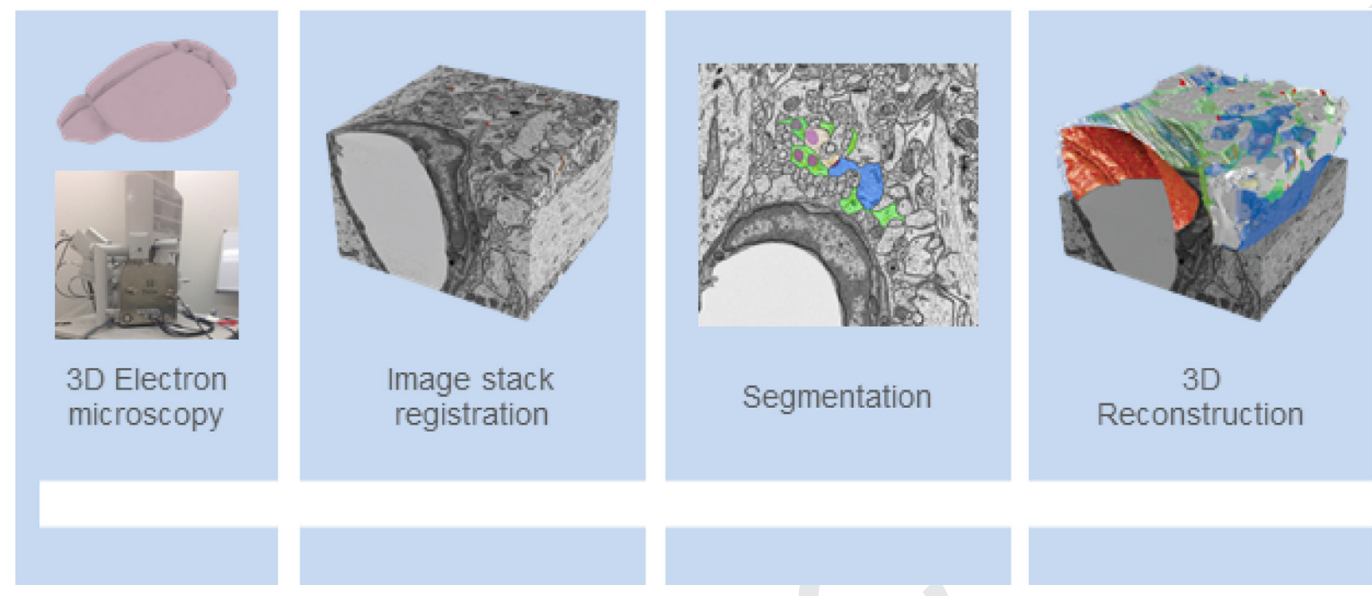

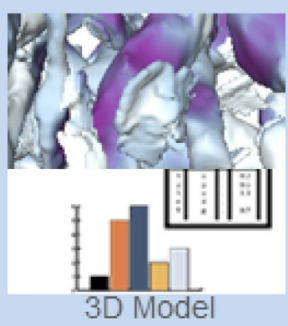

Visualization and Analysis

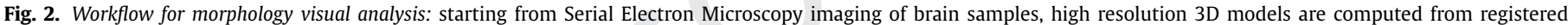
and segmented stacks of images and used as input for visualization and analysis tools.

unknown functions $[3,24]$. This hypothesis illustrates how lactate is delivered to neurons during intense synaptic activity, and moreover glycogen-derived lactate has been related to one of the most important physiological brain functions, memory formation [25]. Despite its importance in both energy metabolism and memory, characterization of brain glycogen distribution has not been fully addressed. Recently, Oe et al. [26] investigated cerebral glycogen in mice using light microscopy and immunohistochemistry (IHC), and they observed a punctate distribution localized predominantly in astrocytic processes. With respect to the intra-cellular distributions, Cali et al. [11] observed that glycogen is localized primarily in small processes and clustered around boutons and spines. More investigation efforts are needed at addressing how other physiological processes other than learning, like for instance aging, are related to modifications in glycogen clustering and how this might be involved in declined memory abilities. Morphological studies are required to evaluate the influence of glycogen distribution compared to the surrounding neuronal structures (dendrites, axons and boutons) under several conditions. We propose here an illustrative analysis model aimed at easing morphological analysis of the distributions of glycogen on high resolution 3D reconstructions from micrometer resolution electron microscopy stacks of rodents brain samples.

\subsection{Morphology visual analysis}

The general workflow for 3D reconstruction and visual analysis of brain morphology (see Fig. 2) begins with sample preparation and 3DEM [27] imaging. Acquisition of biological tissues can be performed automatically at a z-resolution of 5-50 nanometers depending on the cutting technique [27]. After imaging, image stacks needs preprocessing prior 3D reconstruction of the various cellular structures. Notably, one has first to proceed with the registration of the stacks using the Stackreg tool available for ImageJ [28], although this step was more critical when serial sections were obtained manually and then imaged using TEM [29]. Following image registration, the stack can then be segmented, by means of manual or semi-automatic segmentation techniques [30,31]. Automated and semi-automated segmentation techniques reduce tremendously the time and effort to put into the generation of the three-dimensional model, given that the number of sections composing a single image stack could reach thousands with no efforts, as the acquisitions are nowadays fully automated. Nevertheless, an accurate manual proofreading of the segmentation should follow in these cases; depending on the size of the stack and the amount of objects reconstructed, proofreading could be as demanding as a manual 3D reconstruction from scratch. Indeed, computer scientists are putting efforts in improving proofreading strategies [32]. The dataset so obtained includes high-resolution, segmented image stacks that can be visualized, explored and analyzed with a variety of tools based on either volume data representation, or surface mesh generated from the segmentations. Currently many neuroscientists take advantage of commercial or free software solutions [33-35] for 3D segmentation and reconstruction, and develop custom plug-ins for specific statistical and morphometric analysis $[11,13,15,17,26]$. Similarly, tools for visual analysis directly relying on segmented volume data are gaining visibility with the emergent field of connectomics [36]. They are mainly designed to explore the reconstructions of nanoscale neuronal connectivity for the interactive visual analysis using a dynamic-driven query approach [16]. We adapted this framework, designed for connectomics, to speculate on how the intracellular astrocytic distribution of glycogen granules impacts, from a neuroanatomical point of view, on synaptic activity, synapses formation or signal propagation in axons and dendrites. In particular, whereas is common in neuroscience to adapt to astrocytes tools that has been designed 
to characterize neurons, in our case the analytical tool has been designed for the study of astrocytes.

\subsection{Density mapping in $3 D$ structures}

Mapping density distributions to a visual attribute, such as color, is a common practice in visualization. The Allen Brain Atlas viewer [37] maps density and level of gene expression to size in 3D and color respectively. BrainNet Viewer [38] renders a voxel-based network using volume-to-surface mapping. Pycortex [39] uses a pixel-based mapping to render voxels true to form. Goddard et al. [40] describe interactive methods for visualizing density maps using colors and by fitting atomic models. Recently, Agus et al. [41] have shown how density maps can be used to derive points of interest in work of arts in digital museums by tracking the users viewing positions. Our illustrative method is also related to photon map rendering techniques, which have been popularized in ray tracing [42], shadow rendering [43] and global illumination [44]. Importance maps [45] are used to guide the emission of energy packets. The design of our absorption model was based on the method by Agus et al. [41] for deriving focus points and integrated it with a radiance transfer mechanism aimed to compute the influence of glycogen granules to nearby neurites.

\subsection{Visual analysis in stereoscopic environments}

Although most commonly used visualization tools in neuroscience are designed as desktop applications, there is general consensus that the use of stereoscopic techniques can provide a more immersive way to explore brain imaging data [46,47], and that the increased dimensionality provided by stereoscopy is beneficial for understanding depth in the displayed scenery [48,49]. With respect to immersiveness, the effect of stereoscopy have been previously extensively evaluated in the context of visual analysis of volume data, for semitransparent volume rendering [50,51] and isosurface [52], for confocal volume images [53], and for interactive graph analysis $[46,48,54,55]$. Here we report on preliminary results of a user study that we carried out for assessing the effects of stereo cues provided by HMDs during the interactive visual analysis of high-resolution surfaces with color-mapped absorption functions.

\section{Glycogen lactate absorption model}

The proposed illustrative model highlights regions of astrocyteand-neurite membranes where the lactate shuttling is more likely to occur. By considering a photon mapping analogy, we represent glycogen granules as energy sources that emit energy packets, which can be absorbed by the surrounding neurite structures (see Fig. 3), according to constraints derived from the theoretical mechanisms of neuro-glia metabolic coupling $[3,56]$ :

- Power source: the released energy depends on the size of glycogen granules, which are represented as spheres of varying radius (see Fig. 3). According to our illustrative model, these granules cast energy packets onto the surrounding structures, with a number proportional to its size.

- Energy irradiation: a restricted set of neurotransmitters and neuromodulators is able to promote glycogenolysis in astrocytes [3]. They can be modeled as anisotropic directionselective energy irradiation modulators, and included as importance maps [45] for modulating or blocking the energy emitting directions (indicated in red in Fig. 3).

- Energy absorption: the shuttling of derived lactate from astrocyte to neurons is mediated by lactate transporters (MCTs) [57],

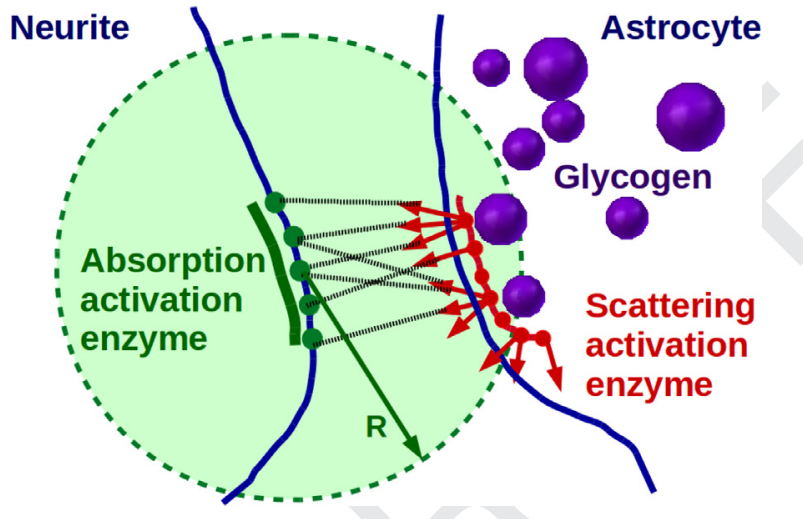

Fig. 3. Glycogen lactate absorption model scheme: energy packets are scattered from glycogen granules according to the size, and presence of activation enzymes. Absorbed energy is computed on per-vertex basis with respect to the presence of absorption enzymes and a distance threshold. (For interpretation of the references to color in this figure, the reader is referred to the web version of this article.)

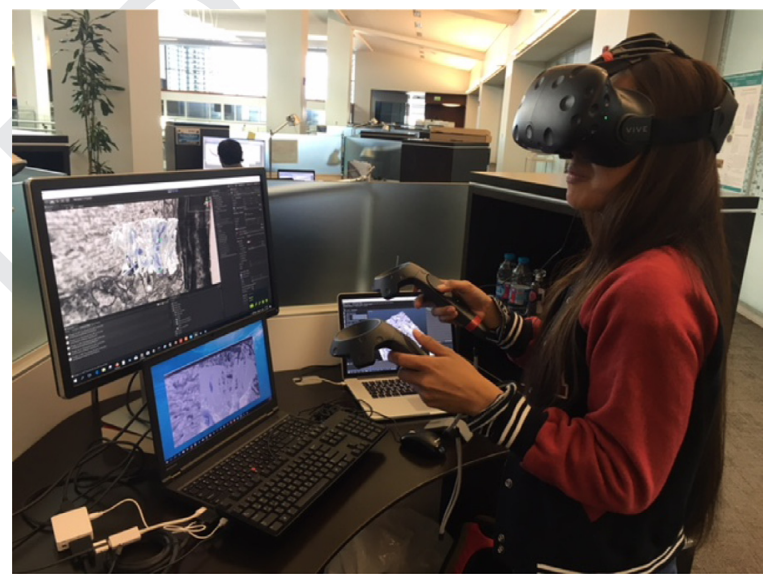

Fig. 4. Immersive environment with $H M D$ : the absorption model has been integrated into an interactive virtual reality environment for real time exploration of morphologies with head mounted display.

which can be modeled as anisotropic direction-selective modulators, for energy packets reaching the neurite surface (indicated in green in Fig. 3).

With respect to the radiance transfer, we considered an absorption model similar to Lambert-Beer law [58] that incorporates a distance-based gaussian decay from each glycogen power source; thereby reflecting the influence of glycogen granules both near and far to membranes. The constraints are incorporated in the model as modulators or switch functions, affecting both the energy absorption and the irradiation decay. Hence, given a neural process at position $\mathbf{x}^{\mathbf{p}}$ and a glycogen granule energy packet $g_{i}$ at position $\mathbf{x}_{\mathbf{i}}^{\mathbf{g}}$, we define an absorption rate as:

$p_{\alpha}\left(\mathbf{x}^{\mathbf{p}}, g_{i}\right)=K\left(r_{i}^{g}\right) \lambda^{g}\left(\mathbf{x}_{\mathbf{i}}^{\mathbf{g}}, \omega_{i}^{p}\right) \lambda^{p}\left(\mathbf{x}^{\mathbf{p}}, \omega_{i}^{p}\right) e^{-\int_{x_{i}^{g}}^{x^{p}} \mu(x) d x}$,

where $K\left(r_{i}^{g}\right)$ is the glycogen irradiation power, $\lambda^{g}$ is a modulating anisotropy term taking into account the presence of activating enzymes for glycogenolysis, $\lambda^{p}$ is a modulating anisotropy term taking into account the presence of enzymes for lactate metabolization, and $\mu(x)$ is the absorption coefficient taking into account the tortuosity for energy transport due to occlusion or friction along the path connecting the glycogen source power to the process. The modulating functions $\lambda^{g}, \lambda^{p}$ depend also on the irradiation direction $\omega_{i}^{p}$. The total energy absorption rate is obtained by summing 
Table 1

Processing statistics: dataset size as bounding box (\# BB) in microns, number of vertices in millions $(V)$, number of glycogen granules (GL), number of emitted $K$ packets $(P)$, and the processing time in minutes.

\begin{tabular}{llllll}
\hline Dataset & $\mathrm{BB}(\mu \mathrm{m})$ & $V(M)$ & $\mathrm{GL}$ & $\# P(K)$ & $T(\mathrm{~m})$ \\
\hline Hippocampus1 & $7.1 \times 6.7 \times 4.7$ & 6.28 & 1007 & 135 & 15.8 \\
Hippocampus2 & $14 \times 14 \times 24$ & 0.98 & 9438 & $38 \mathrm{~K}$ & 25.3 \\
Cortex1 & $5 \times 5 \times 5$ & 0.66 & 3037 & 407 & 13.8 \\
Cortex2 & $5 \times 5 \times 5$ & 1.08 & 7719 & 110 & 2.8 \\
Cortex3 & $5 \times 5 \times 5.9$ & 1.46 & 4128 & 546 & 5.7 \\
Cortex4 & $5 \times 5 \times 5$ & 2.78 & 7048 & 110 & 6.8 \\
\hline
\end{tabular}

up the contribution of all glycogen energy packets:

$p_{\alpha}\left(\mathbf{x}^{\mathbf{p}}\right)=\sum_{i} p_{\alpha}\left(\mathbf{x}^{\mathbf{p}}, g_{i}\right)$.

The obtained absorption rate provide a local approximation of the quantity of lactate absorbed in the unity of time.

\section{Implementation}

We implemented GLAM model in C++. Given the limited information of spatial distribution of lactate membrane transporters (MCTs) to date, an accurate absorption model cannot be computed. We hence simplified our working hypothesis, and considered as isotropic the emission from granules sources, the absorption from the neural structures of interest, and the tortuosity along the path connecting the neurite to the structure of interest. As a consequence, energy packets can be generated uniformly on the surface of the glycogen granule, since glycogen granules irradiate in same way along all directions, and stored in a kd-tree [59]. The number of generated photons is proportional to the size of glycogen granules and hence to the number of glucose molecules; this is in accordance to the biology of the system, as the size of the granules varies depending on their glucose content, and therefore bigger granules can store more energy [8,9]. Photons were chosen to be ranging from $5 \mathrm{~K}$ to $50 \mathrm{~K}$ and to the number of lactate molecules generated. The absorption rate computation is then performed on a vertex basis, and accounts for the K-nearest-neighbor (KNN) search distance threshold $\mathrm{R}$ by presuming that distant energy packets do not significantly contribute to the absorbed energy of structures (indicated in green in Fig. 3). This distance is chosen in a way to be a trade-off between computational workload and the mean length of peripheral astrocytic processes [60] (less than $1 \mu \mathrm{m}$ ). Given the isotropic assumption, both the modulating functions and absorption coefficient become constant, so Eq. (2) is simplified to

$p_{\alpha}\left(\mathbf{x}^{\mathbf{p}}\right)=\sum_{i} K_{i} \lambda^{g} \lambda^{p} e^{-\mu\left\|\mathbf{x}_{\mathbf{i}}^{\mathbf{g}}-\mathbf{x}^{\mathbf{p}}\right\|}$,

and computed by accumulating all contributions from energy packets in the surrounding area. For exploration and analysis, the computed absorption rates on the datasets of Table 1 can be colormapped with respect to a user-defined color scheme. In the results shown in this paper, we consider a log-normalized color mapping performed according to a sequential Color Brewer scheme BuPu [61] (see an example in Fig. 5).

\subsection{Visual hints}

In order to provide additional clues for interpreting main absorption patterns, we considered two additional visual hints for representing both the peak values and the correlation of the absorption value compared to the exchange surface (i.e. the plasma membrane of the cell). In particular, to highlight the absorption peaks, we relied on a thresholded segmentation of the local absorption value; all vertices above a given threshold were clustered using DBSCAN algorithm [62]. The threshold was chosen as a percent of the maximum absorption value (for most datasets we had $\alpha_{\max }=2500$ and we chose a threshold $\tau=500$ for selecting the highest possible number of local absorption peaks). Then, all clusters were processed to be visualized as spheres onto the neurites meshes: information about max absorption value was color-coded (in all figures the classic colorjet colormap ranging from blue to red was considered), while the spatial extent of the peak was multiplied by the average absorption value and visually represented as the radius of the same sphere (see an example in Fig. 13). This colormap was chosen to better attract the focus of user during exploration over the more intense peaks. Furthermore, to better rank neurites based on individual absorption patterns, we took into account the relationship between the the local absorption values and the surfaces, and we derived an indicator of expected absorption $\langle\alpha\rangle$ for a given neural process, which is the the weighted absorption with respect to the surface:

$\langle\alpha\rangle=\frac{\int_{S} \alpha(\sigma) d \sigma}{\int_{S} d \sigma}$

The expected absorption indicator can be used to filter out those objects whose expected absorption was below a certain threshold, and for ranking neural objects. In the rest of the paper, the expected absorption indicator will be used for color-mapping objects with respect to a sequential sequential Color Brewer scheme [61] YlOrRd ranging from light yellow to dark orange (see an example in Fig. 15). The color-mapped objects can be integrated in a general 3D exploration analysis framework considering surface representations. In our case, we tested a desktop solution by considering a typical analysis framework used by neuroscientists. The solution consists of NeuroMorph analysis toolset inside the Blender environment [13], and enables domain scientists to perform visual correspondences between reconstructed objects and the original EM stacks.

\subsection{Virtual reality display setups}

On the basis of the original standard desktop setup, two different virtual reality systems were developed to test whether the proposed model can simplify visual analysis. For engaging collaborative discussions, we exploited the capabilities of CAVE (cave automatic virtual environment) [63], whose space is a cubic room with 3-m sides that are each projected with two 8-megapixel streams. With this setup, several people are able to simultaneously stand inside a 3D model, where details could be magnified on demand and eventually become a million-fold larger ( $\mu \mathrm{m}$ to $\mathrm{m}$ ). The 3D model is projected directly from the 3D modeling Blender window interface by using TechViz software (http://www.TechViz.net), while the user could manipulate the model inside the room with a controller. A head-tracking system modify the perspective of the object based on the position of the user, who is able to move freely in the room and observe the model from different positions. Furthermore, in order to provide also a lightweight and effective solution for interactive exploration and visual analysis of the models and the EM stack, we took advantage of a portable immersive environment by using a low-delay headset. For this test case, we used the HTC Vive, which carries 70 sensors, and provides $360^{\circ}$ headtracking with a refresh rate of $90 \mathrm{~Hz}$ [64]. We then designed and developed a virtual environment, within the Unity framework. It provides users with the capabilities of loading and interactively select 3D neurite models with the GLAM absorption map textured on the surfaces, while interactively scrolling the EM image stack used for the segmentation, and exploring it in 3D to perform correlative visual analysis in a straightforward way (see Fig. 4). The executable 

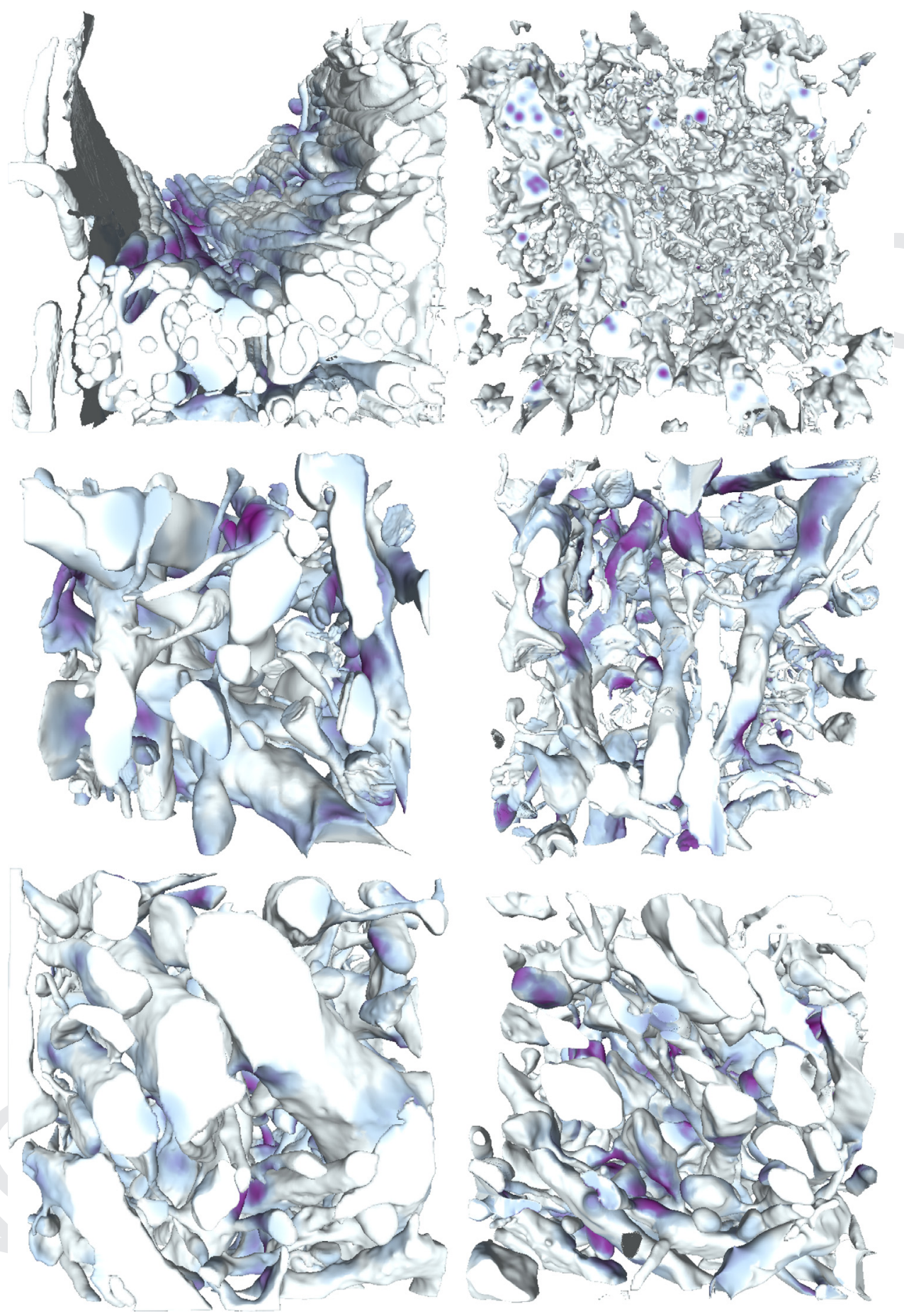

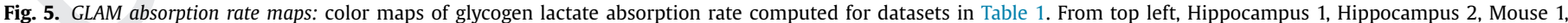
Mouse 2, Mouse 3, Mouse 4. Images produced with Meshlab software [66].

of our Unity-based VR application for real time exploration with HTC Vive, together with example data, are deposited in a Dryad repository [65]. Finally, the accompanying video shows virtual exploration sessions performed both in large-scale CAVE system and with Head-Mounted display (Supplementary Video 1).

\section{Results}

We tested the GLAM absorption model on a series of high resolution 3D reconstructions from rodents brains, and in different setups. Datasets include neural processes, like dendrites, ax- ons, and astrocytes, as well as organelles like boutons and spines. Table 1 provides statistics on data processing, with respect to the model size in terms of bounding box, number of vertices, number of glycogen granules, number of emitted energy packets, and processing time in minutes. For all datasets, we considered $R=0.5 \mu \mathrm{m}$ as influence radius for accumulating energy packets contributions and processing times were computed on a laptop equipped with Intel i7-4700HQ 2.4 GHz CPU and $8 \mathrm{~GB}$ RAM and running Linux Ubuntu kernel 3.13. The appearance of full color-mapped datasets is represented in Fig. 5. The GLAM model is currently used for investigating potential lactate absorption patterns in $3 \mathrm{D}$ brain struc-
403 404 405 406 407 408 409 410 411 412 413 


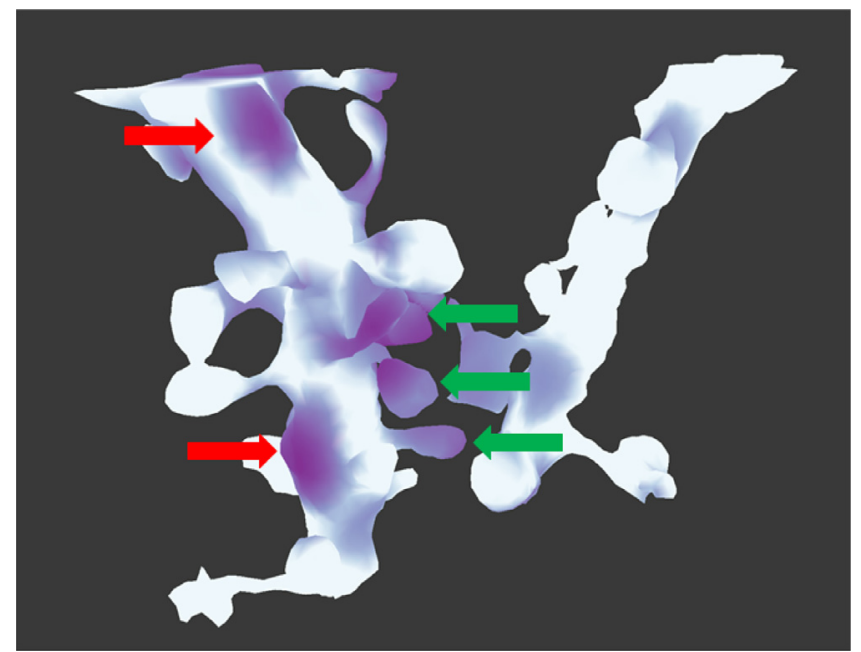

Fig. 6. Synaptic plasticity: the analysis of absorption map highlighted the presence of shaft synapses, from which spines can generate (red arrows), and highly plastic small-size dendritic spines (green arrows). Dendrite detail from dataset Hippocampus1, rendered within Blender environment. (For interpretation of the references to color in this figure legend, the reader is referred to the web version of this article.)

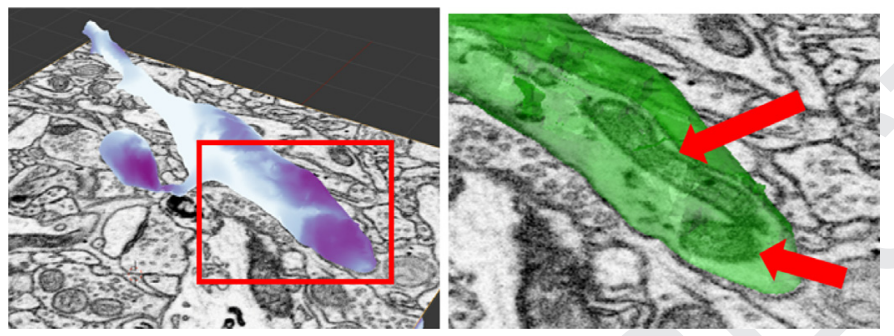

Fig. 7. Proximity with mitochondria: the peaks of glycogen lactate absorption maps (left) can highlight proximity with respect to mitochondria (red arrows on the right). Dendrite detail from dataset Somatosensory Cortex1. Images produced with Blender and Neuromorph softwares [13]. (For interpretation of the references to color in this figure legend, the reader is referred to the web version of this article.)
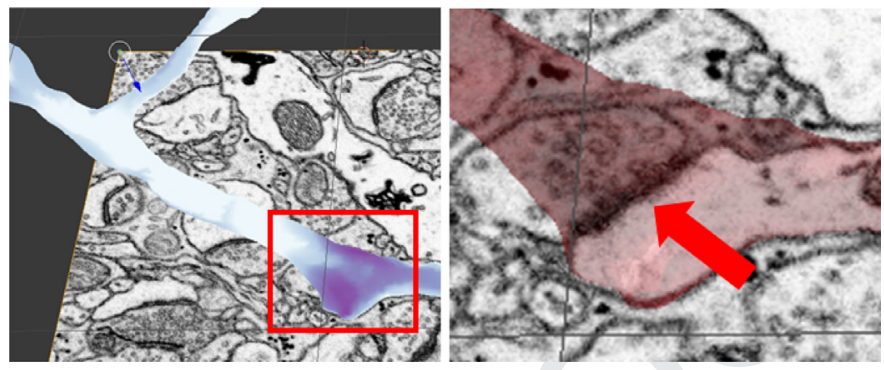

Fig. 8. Proximity with synapse: the peaks of glycogen lactate absorption maps (left) are close to a shaft synapse (red arrow on the right). Axon detail from dataset Somatosensory Cortex2. Images produced with Blender and Neuromorph softwares [13]. (For interpretation of the references to color in this figure legend, the reader is referred to the web version of this article.)
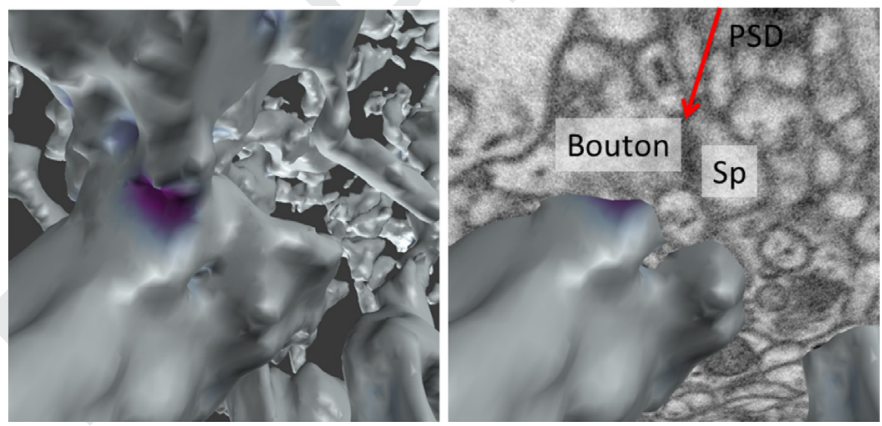

Fig. 9. Proximity with PSD: the peak of glycogen lactate absorption map on astrocyte morphology is close to a PSD and a bouton (on the right). Astrocyte detail from dataset Hippocampus2. Images produced with Blender and Neuromorph softwares [13].

to dendritic mitochondria (red arrows on the right), which are the intracellular organelles responsible for the usage of lactate to ultimately synthesize ATP [57];

- Synapses: Fig. 8 shows that the glycogen absorption map peaks (on the left) show a spatial correspondence with respect to an asymmetric (presumably excitatory) shaft synapse; a continuous neurotransmitter release at this site might result in the formation of a spine;

- Boutons and PSDs: Fig. 9 shows another example highlighting that absorption map peaks on astrocyte morphology can be associated with the presence of boutons, spines and PSDs, further indicating that glycogen generated energy is fundamental for sustaining neural connections and spines generation;

- Axons: Fig. 10 highlights absorption peaks in specific axon bundles in dataset Hippocampus1, conforming the hypothesis that axons uptake glycogen-generated lactate $[68,69]$.

- Lactate channels: in Fig. 12, a detail of dataset Hippocampus 2 shows how absorption maps are used for the analysis of glycogen distribution inside astrocyte morphologies, in order to speculate about the possible spatial distribution of the channels for lactate emission.

These examples show how the absorption model can be successfully exploited to test neuroenergetics hypotheses and rapidly investigate the spatial correspondence with specific features on morphologies. In general, domain scientists found absorption maps particularly useful during glycogen analysis for the spatial discrimination capabilities and for reducing clutter in specific cases where glycogen granules can occlude the features of interests, since the model is able to provide indication of spatial distribution directly mapped in the structure of interest (see Figs. 11 and 12). 


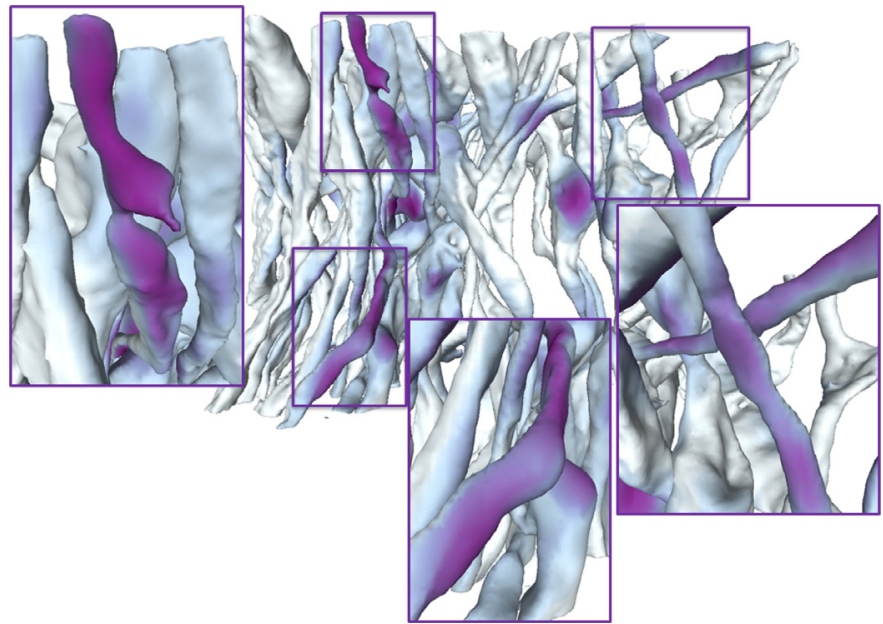

Fig. 10. Proximity with axons: the peaks of absorption map show that specific bundle of axons exhibit high lactate absorption rate. Axons details from dataset Hippocampus 1. Images produced with Meshlab software [66].
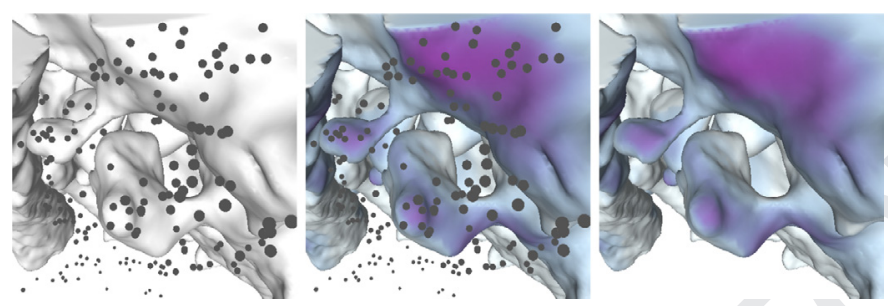

Fig. 11. Glycogen analysis: domain scientists perform analysis of glycogen distribution compared to neuron morphologies (left). Absorption maps help analysis in spatial discrimination (center), and in some cases can reduce clutter due to glycogen granules overlapping over the regions of interest (right). Dendrite detail from dataset Hippocampus1. Images produced with Meshlab software [66].
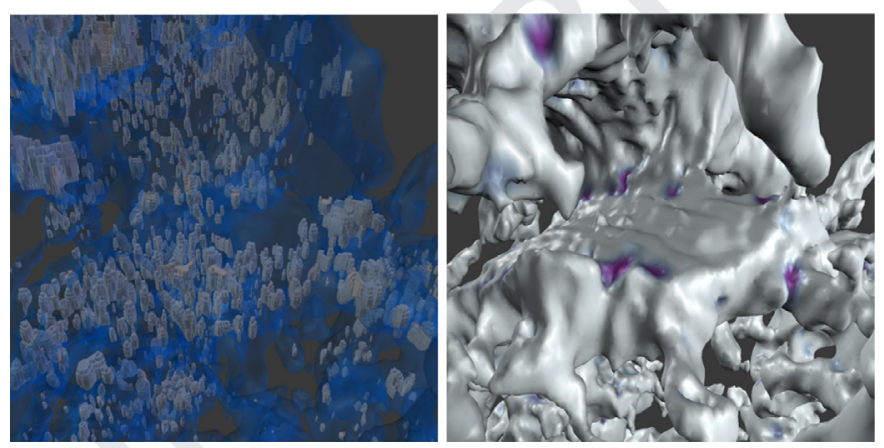

Fig. 12. Astrocyte glycogen analysis. Left: glycogen analysis in astrocyte is particularly complex since transparent morphologies can clutter and reduce spatial discrimination. Absorption maps in this case can help in the analysis of specific morphology details, since the information of the glycogen distribution is directly mapped on the surface of the structure. Right: absorption map in astrocyte surface provide indication of the most probable channels for lactate shuttling. Dataset Hippocampus2 rendered inside Blender environment.

\subsubsection{Planning of sparse reconstructions}

The absorption model and the peak spherical markers can provide tools for planning sparse reconstructions on big image stacks. Given a reconstructed astrocytic process and its glycogen granules, the GLAM model can be used to locate sites where the lactate shuttling is most likely to occur, while the colored spherical markers provide hints for ranking the local peaks. Domain scientists can take advantage of this tool to pre-select regions and elements of

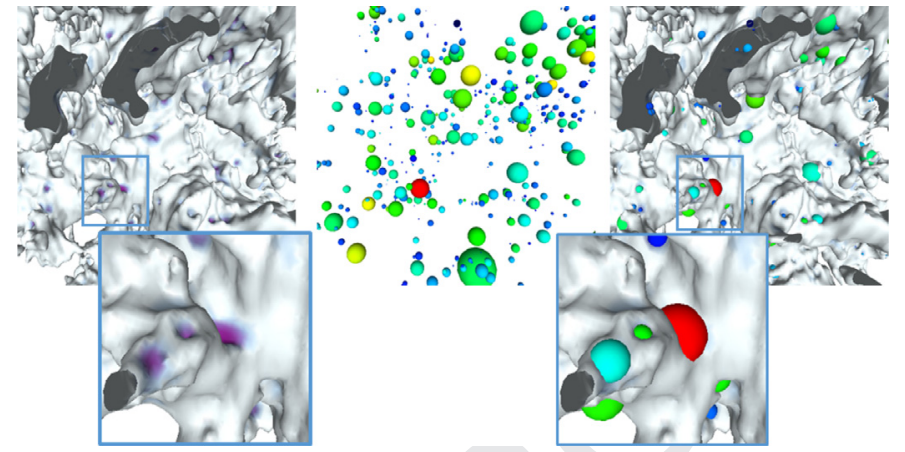

Fig. 13. Reconstruction planning: domain scientists can plan sparse reconstructions from preliminary labeling, by using GLAM to highlight absorption peaks. Left: absorption map of dataset Hippocampus 1. Center: absorption peak clusters represented as colored spheres of different dimensions. Right: mapping of absorption peak clusters over the astrocyte reconstruction. Images produced using Meshlab software [66]. (For interpretation of the references to color in this figure, the reader is referred to the web version of this article.)

the stack that are worth reconstructing in 3D, and discard uninteresting areas that might increase both occlusion without adding relevant information and processing time to produce a dense segmentation. As example, Fig. 13 shows the GLAM model computed on the astrocyte in dataset Hippocampus 2, together to the colored absorption peaks, highlighting the priority areas. The technique is currently used for performing analysis on image stacks recently acquired using block-face electron microscopy from different cohorts of mice, with the target of investigating the glycogen distribution under different network activation states (i.e. naive versus learning mice) and/or impaired glycogen metabolism using the glycogen synthase inhibitor DAB. In the specific context of this project, the use of the large scale VR environment improved the usefulness of 3D objects, since domain scientists can make collaborative analysis and take decisions on how to conduct future segmentations. As example, Fig. 14 shows interactive sessions of exploratory analysis of Hippocampus 2 model together with EM slices rendered with NeuroMorph package [13]. In this case, the peaks in absorption maps over the astrocyte morphology drove domain experts in discussion and comparison for confirming and formulating hypothesis on lactate shuttling mechanisms and decide which areas of the stacks are worth to be reconstructed for successive analysis.

\subsection{Test cases}

The GLAM model was successfully used for specific exploratory and comparative analysis targeted to highlight different conditions in absorption patterns.

\subsubsection{Analysis of hippocampus stack}

The GLAM maps, together with absorption peak representations and expected absorption indicators were used for a detailed visual analysis of a dense reconstruction from rat hippocampus. Detailed information can be found in [11]. Briefly, the dataset contains a dense reconstruction obtained using a combination of iLastik and TrakEM2 software, starting from an isotropic $(6 \mathrm{~nm})$ image stack from a 220 cubic micrometers volume of dorsal hippocampus. That dataset has been used to describe from a qualitative and quantitative point of view the distribution of astrocytic glycogen granules compared to pre o postsynaptic targets, based on a simple nearestneighbor algorithm adapted for a 3D dataset. According to a standard classification of the neurites, neuroscientists were interested to understand the absorption patterns and to compare the different neurites with respect to it. Fig. 15 shows results of classification of various neurites with respect to GLAM absorption map together 

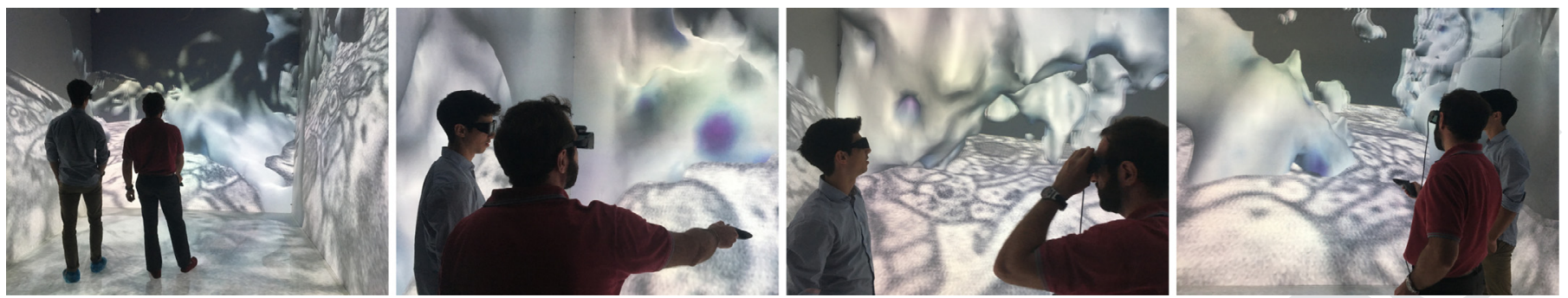

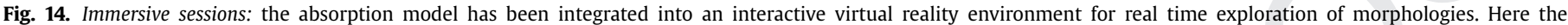
exploration of astrocyte morphology of dataset Hippocampus 2 is shown.
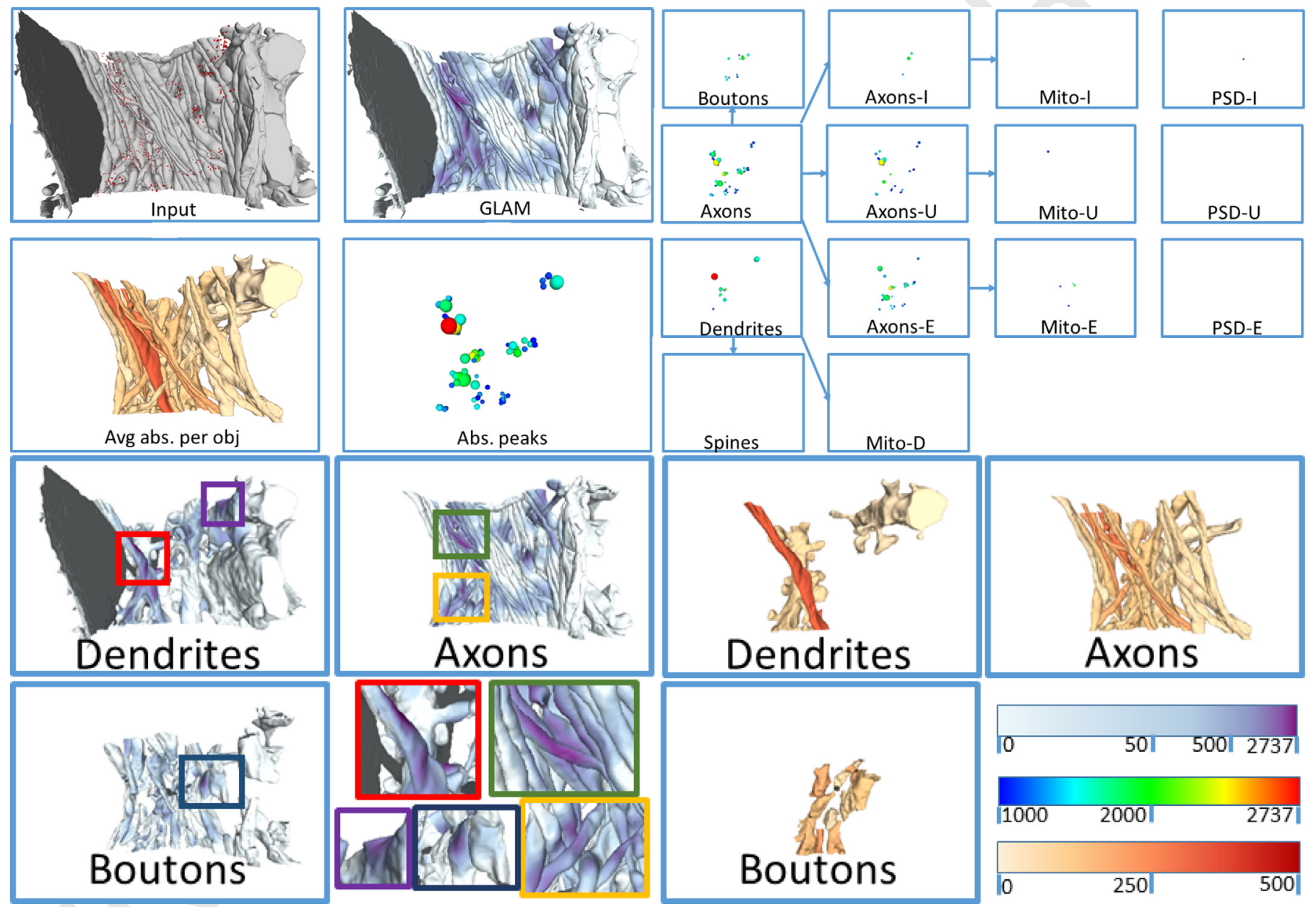

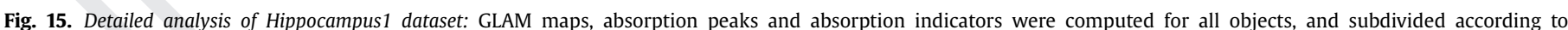

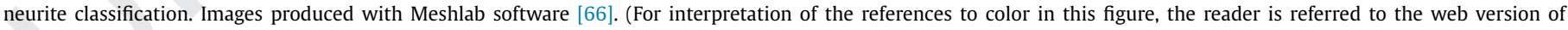
this article.)

with peak and expected absorption indicators: specifically the objects were classified as axons, dendrites and postsynaptic densities (PSD). Axons whose boutons were present and labelled have been further classified as excitatory (Axons-E), inibitory (Axons-I) and unknown (Axons-E) axons, together with the respective mitochondria (Mito-I, Mito-U, and Mito-E). Similarly, spines were marked within dendrites, and their mitochondria was reconstructed (MitoD); PSDs were also classified as excitatory (PSD-E), inibitory (PSDI), and unknown (PSD-U).

For the neurites involved it is shown the GLAM absorption map, the absorption peak spherical markers, and the filtered objects color mapped against the expected absorption value (see Eq. (4)). A 527 preliminary visual investigation of absorption peaks (in Fig. 15 top 528 right) showed no significant absorption in PSDs and in spines. In- 529 stead, it seemed that the main glycogen-derived activity was fo- 530 cused on axons (in particular in excitatory and unknown ones - 531 green and orange box in Fig. 15 bottom left), in a subset of ex- 532 citatory boutons (blue box in Fig. 15), and in particular dendrites 533 with immature spines (red and purple box in Fig. 15). Interest- 534 ingly, these observations are in line with the findings of the orig- 535 inal work [11]. In order to further investigate these objects, they 536 were filtered with respect to the mean absorption indicator (in 537 


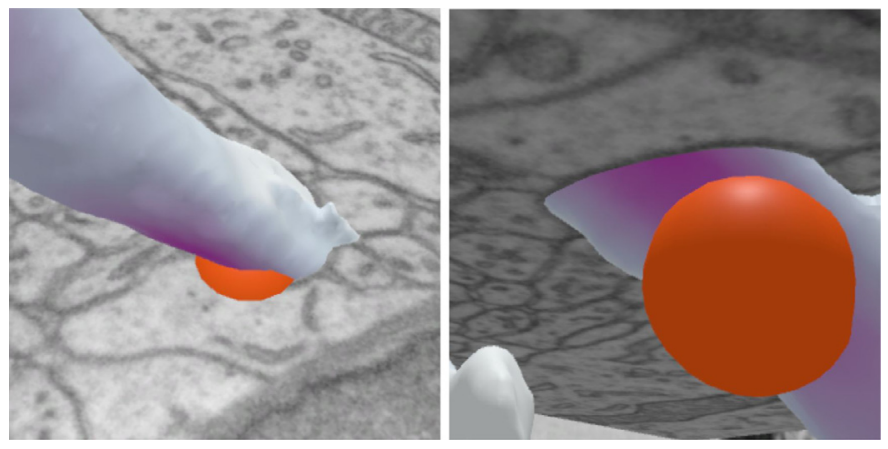

Fig. 16. Analysis of dendrite main peak on Hippocampus1 dataset: a comparative analysis with EM stack of the main peak in the Hippocampus1 dataset did not reveal a significant proximity of mitochondria and synapses. Images produced with the HTC Vive Unity application.

Fig. 15 bottom right), where it appeared that a specific dendrite with immature spines showed a particular intense absorption pattern (see red box highlight in Fig. 15 bottom left.) That dendrite was then carefully examined with the VR HMD system, especially in the area of the most intense peak, together with the original EM stack, in order to find presence of eventual synapses or mitochondria in the close neighborhood (see Fig. 16). Since no synapses or mitochondria were identified, neuroscientists concluded that lactate shuttling (or glycogen accumulation) in that area is due to other reasons that need to be explored.

\subsubsection{Analysis of somatosensory cortex datasets}

Domain scientists carried out also a detailed visual analysis on densely reconstructed somatosensory cortices from a cohort of adults and aged mice. Datasets Mouse1, Mouse2, Mouse3, Mouse4 are part of a series of datasets used to analyze the effects of aging on the neuropil structure. In particular, Mouse1 and Mouse3 are adult mice, while Mouse 2 and Mouse 4 are geriatric animals. In this case, neurites were classified as axons, dendrites, boutons, spines, and PSDs. Fig. 17 show GLAM maps (on top), absorption peaks (in the middle), and the filtered expected absorption (in the bottom). In this case, considering either the absorption peaks and the expected absorption images, domain scientists were able to recognize similarity in patterns between Mouse 1 and Mouse3, and between Mouse 2 and Mouse 4 (see red boxes in Fig. 17). In general, samples taken by older animals are characterized by a more evenly distributed glycogen-derived influence compared to younger ones, suggesting that glycogen is clustering preferentially around specific sites in younger brains. Overall, domain scientists were able to highlight that the peaks are preferentially focused around axons and presynaptic boutons (see blue boxes in Fig. 17). Finally, Post-synaptically only specific spines and PSDs show a significant signal; interestingly, the smaller ones (supposedly more involved in plasticity) are more absorptive compared to the big ones (see green boxes in Fig. 17). In conclusion, domain experts confirmed preliminary observation made on this dataset, and noted interesting similarities with the rat hippocampus dataset, suggesting a non random distribution of the cloud of glycogen points not only between different brain regions, but also between different species.

\subsection{User study: comparison between desktop and VR solution}

The use of Virtual Reality in Neuroscience is still at its early stages [11], therefore the design of user studies for evaluating the performance of virtual environments for analysis tasks had to be designed almost from scratch, because of the lack of standardized guidelines [46]. Hence, we decided to rely on typical Virtual Re- ality Assessment Methodologies [70] and conducted a user study aimed at evaluating the benefits in the use of VR technologies, in the context of the GLAM model. To this end we analyzed two visualization setups, a desktop system and an HTC vive Head-Mounted Display (HMD) setup, and made a quantitative comparison between stereoscopic and monoscopic viewer use. Users were asked to fill in a system usability scale questionnaire (SUS) [71] after each training session. That is, one after using the monoscopic view desktop application, and one after using the stereoscopic view VR application (HMD). Furthermore, we collected users feedback by means of open-ended questions. The VR system allowed to visualize the GLAM models together with the superimposed EM micrographs, and an interface for scrolling EM stacks; the standard desktop solution was equipped with Blender to visualize the GLAM models and Neuromorph to interact with it. The study took place in the visualization lab facility of King Abdullah University of Science and Technology.

\subsubsection{Design and protocol}

The study was performed on a group of 27 volunteer staff members. The participants were a mixture of senior scientists and research staff with diverse experience in neuroscience, technology and VR research. To make sure the design of the study was consistent, a pilot study was conducted beforehand. This helped us to guarantee the availability and functionality of the facilities needed as well as remove any factors that may lead to biased results. This first, pilot stage involved 6 participants [72]. The protocol carried on in the formal study was as follows: a short introduction on the GLAM model was explained to the participants, followed by a demonstration on how to use our software tools. Our software applications ran on two different setups, therefore each participant went through two designed sessions: a Desktop application (Blender and Neuromorph) running in monoscopic view, and a Unity-based application using HTC vive HMD running in stereoscopic view. The demo used in the introduction was implemented using a simple use case scenario. Later on, participants proceeded on their own. Users were asked to perform two tasks, involving the GLAM model:

- to count the peaks on a given 3D absorption model of a neuronal cell;

- to select any peak on the given 3D model and perform preliminary analysis using the corresponding 2D EM slice (in other words, to try to find spatial correspondences between the peak and the surrounding cellular structures observed on the EM slice).

We chose Hippocampus 1 stack as support dataset, and five 3D models from the same dataset as test objects. During each session, 3D models were picked randomly for each system setup for each participant, who answered afterwards a 9-item questionnaire derived from [71] ( see Table 2); participants were asked to give scores on a Likert scale of 1-5, with: $1=$ strongly disagree and $5=$ strongly agree. The total time required for both sessions was approximately between 30 and 40 min per participant. Think-outloud comments were also recorded during sessions.

\subsubsection{Discussion}

Results of the 9-item questionnaire are shown in Table 2 in the form of statistics (mean scores, variance and results of ANOVA). The Likert scores are also represented as compact box plots in Fig. 18 (in red the scores for the VR Vive setup, in blue the results for desktop setup). The bottom and top of each box are the first and third quartiles, the black dot inside the box is the second quartile (the median), and the ends of the whiskers extending vertically from the boxes represent the lowest datum still within 
Mouse1
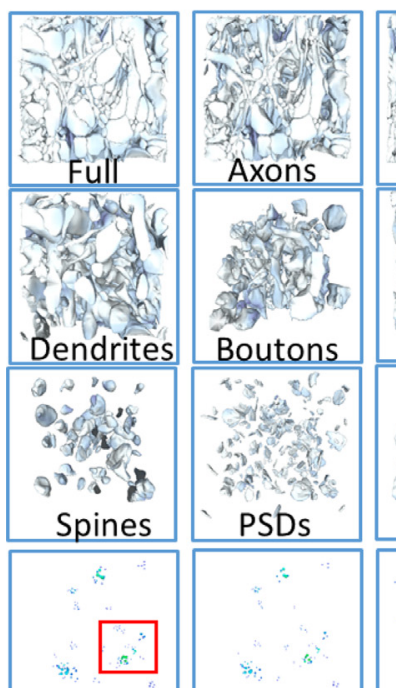

Mouse2
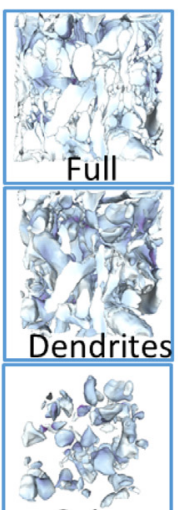

Spines

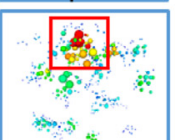

$\rightarrow$ it

Full

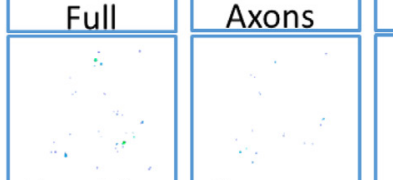

\begin{tabular}{|c||c|}
\hline Dendrites & Boutons \\
\hline Spines & PSDs \\
\hline
\end{tabular}
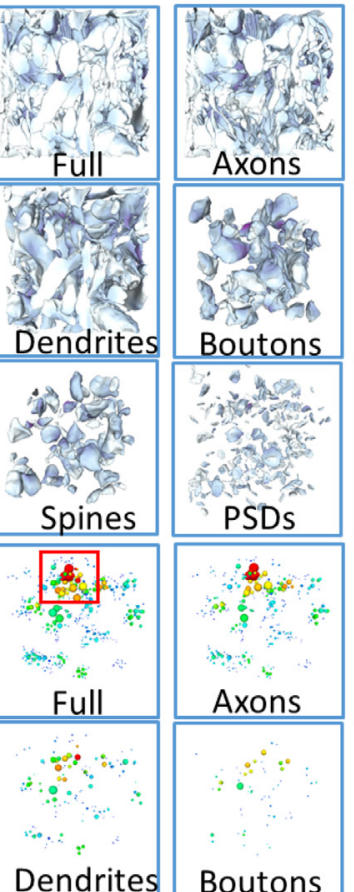

Boutons
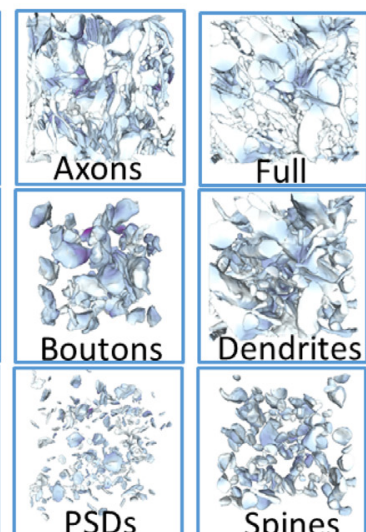

Mouse3

Mouse4

\section{8}
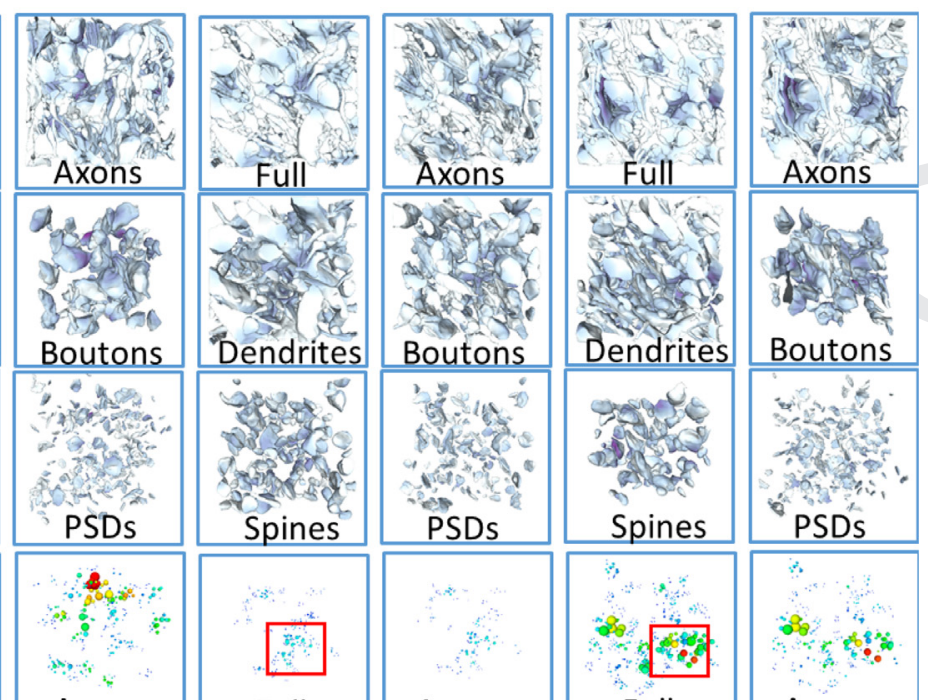

Axons
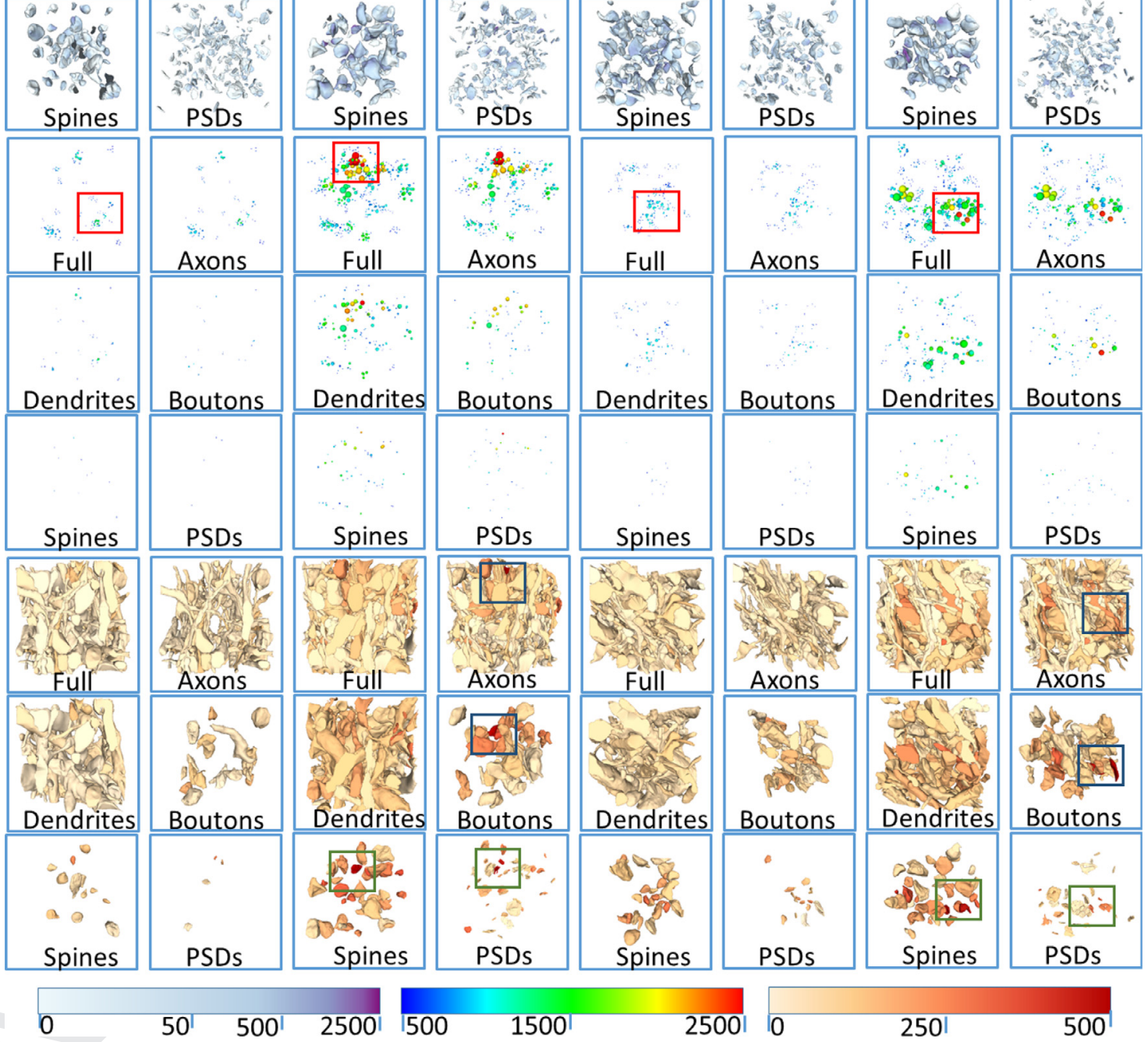

1500

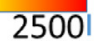

0

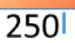

500

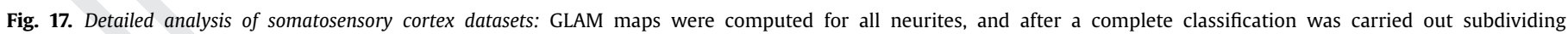

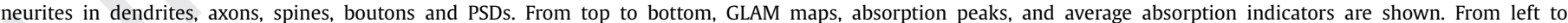

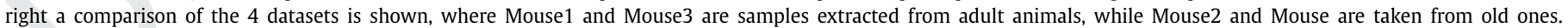
Images produced with Meshlab software [66]. (For interpretation of the references to color in this figure, the reader is referred to the web version of this article.)

1.5 IQR (inter-quartile range) of the lower quartile, and the highest datum still within 1.5 IQR of the upper quartile. Outliers are indicated as small circles. From the scores, it is evident that the VR solution appears, at least, as useful as the desktop solution. In general, scores for all questions show a slight trend towards the VR solution, which becomes significant for question Q4 (users appear to appreciate the 3D interface for exploring color-mapped data), question Q6 (user appear to appreciate the immersivity of virtual environments), and question Q9 (exploratory tasks appear to be easier in a VR setup). However, think-a-loud comments highlighted the typical issues normally encountered when interact- ing with virtual environments (lost in space sensation, and motion sickness and fatigue during long sessions). Finally, feedbacks from expert domain scientists highlighted that they would use the tool regularly for specific targeted examinations, but not for the standard analysis (for example, the peak counting task was perceived easier with the standard Desktop solution). On the other hand, when focused explorations are needed, the VR solution appeared to be more appealing since the stereo 3D natural interface enables users to observe the objects and the correspondent EM stacks at a perceived higher resolution and with better spatial discrimination. 
Table 2

User evaluation: 27 subjects were asked to compare two different versions (VR employing HTC Vive and Desktop) of the interactive system employing GLAM. Table shows the results of the answers of a 9-item questionnaire [71] on a Likert scale ( $1=$ disagree,5=agree), together with a statistical analysis.

\begin{tabular}{|c|c|c|c|c|c|c|}
\hline \multirow[t]{3}{*}{ Question } & \multicolumn{6}{|c|}{ Results (Likert scale: $1=$ disagree $5=$ agree) } \\
\hline & \multicolumn{2}{|c|}{ VR - HTC } & \multicolumn{2}{|c|}{ Desktop - Mono } & \multicolumn{2}{|l|}{ ANOVA } \\
\hline & Avg & $\sigma$ & Avg & $\sigma$ & $F(2,27)$ & $\mathrm{p}$ \\
\hline Q1: I think that I would like to use this application frequently. & 4.384 & 0.726 & 4.115 & 1.066 & 1.48 & 0.235 \\
\hline Q2: I found the application unnecessarily complex. & 1.923 & 1.274 & 1.654 & 0.955 & 1.204 & 0.283 \\
\hline Q3: I think that I would need technical support for using this application. & 2.539 & 1.539 & 2.308 & 1.341 & 1.696 & 0.204 \\
\hline Q4: I like using the interface of this application. & 4.345 & 0.555 & 3.846 & 1.655 & 4.684 & 0.04 \\
\hline Q5: I think that most people would learn to use this application quickly. & 4.115 & 0.746 & 3.731 & 1.325 & 2.617 & 0.118 \\
\hline Q6: I felt very confident using the application. & 4.154 & 0.855 & 3.692 & 1.101 & 5.294 & 0.03 \\
\hline Q7: I needed to learn a lot of things before I could use this application. & 2.115 & 1.306 & 2.231 & 2.025 & 0.355 & 0.556 \\
\hline Q8: The information provided by the application was clear and helpful. & 4.039 & 0.912 & 3.769 & 1.545 & 1.093 & 0.306 \\
\hline Q9: I felt it difficult to interact and control the system. & 1.923 & 0.954 & 2.500 & 2.180 & 4.851 & 0.037 \\
\hline
\end{tabular}

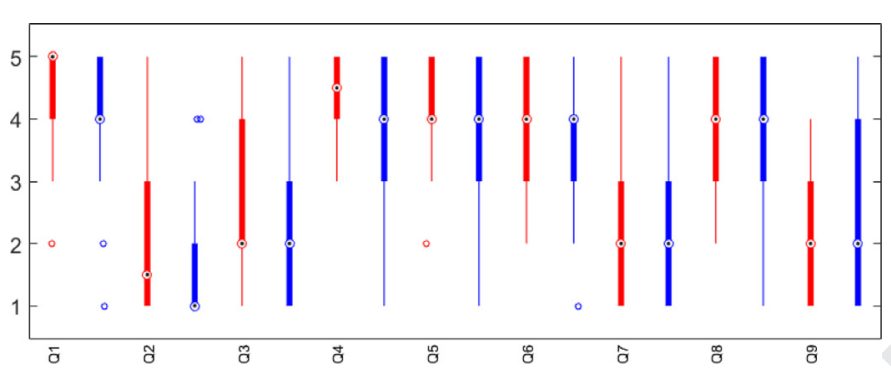

Fig. 18. Comparison desktop vs VR (Vive): box-plots show the comparison between the Likert scores to the usability questionnaire in Table 2 provided to subjects. In red we indicate the scores obtained with the VR solution, in blue the score for the desktop setup. (For interpretation of the references to color in this figure legend, the reader is referred to the web version of this article.)

\section{Conclusions}

We presented an illustrative model of glycogen-related lactate absorption targeted at visual investigation of neural morphology obtained by 3D reconstruction from Serial Block Face Electron Microscopy stacks. The main use of the model is for hypothesis testing of lactate utilization, and to understand whether difference in energy consumption exist for instance among conditions such as normal brain functioning, aglycemia and during memory formation. The model has been integrated in visual frameworks for real time exploration of surface representations in both desktop and Virtual Reality setups. The GLAM model has been successfully used by domain scientists for visual analysis in a number of test cases, and a user evaluation have been conducted to assess the performance of the model in the context of virtual environments. The outcomes of the user evaluation are that VR systems can help in visual analysis for highlighting and focusing in specific details of the neural structure. Information about the cellular and molecular machinery are still needed to precisely model the lactate transport accurately; for instance, the spatial distribution of lactate transporters at nanometric scale needs to be investigated, and its lacking limits the use of GLAM model as a predictive analysis tool of lactate absorption. However, given the encouraging results of our preliminary evaluation, we plan to incorporate more precise scattering and absorption constraints by processing data coming from in-vivo and in-vitro lactate transporters acquisition [73]. Specifically, an anisotropic description of the lactate transport could be obtained by modeling constraints as function of tissue density. We plan to explore this extension for performing volumetric absorption computation directly on Electron Microscopy Stacks and visualize it using volume rendering techniques. Furthermore, the glycogen lactate absorption computation is currently integrated into a visual analysis workflow for morphology investigations [13]. However, a limitation of the whole pipeline is that it requires a previously reconstructed 3D model, which takes a considerable amount of time and effort to process. Despite that, we believe that the pattern of glycogen distribution can provide significant cues for morphology discrimination, and that the usage of an irradiation energy scheme can provide hints for visual understanding of neural morphology evolution. We plan to integrate the absorption model in systems for directly rendering raw SEM image stacks [74] and in systems for real time exploration of abstract representations of brain structures [17]. Finally, the positive outcomes of the user evaluation for the VR solution make us believe that Virtual Reality, a technology that is exploding thanks to gaming industry, and making its way into life science and medical sciences, have the potential to support neuroanatomical and neuronergetics investigations. Custom tools taking advantage of visualization methods, visual analytics technologies and particular interaction metaphors can provide added values to domain scientists analysis. We plan to further explore this field in future.

\section{Acknowledgments}

We thank Kalpana Kare for the technical support for the 3D reconstruction pipelines and coding; the KVL team for the support in the use of CAVE and for providing computing clusters to process the image stacks for 3D reconstructions; Graham Knott and the BioEM Facility at EPFL (Lausanne, Switzerland), and Elena Vezzoli from University of Milano (Milano, Italy), for providing the EM stacks that we used for extracting the 3D models. This work was supported by the KAUST Grant KAUST-EPFL Alliance for Integrative Modeling of Brain Energy Metabolism to Pierre Magistretti and Henry Markram.

\section{Supplementary material}

Supplementary material associated with this article can be found, in the online version, at doi:10.1016/j.cag.2018.04.007.

\section{References}

[1] Verkhratsky A, Butt A. Astrocytes. John Wiley, Ltd; 2007. p. 93-123. ISBN 9780470517796.

[2] Somjen GG. Nervenkitt: notes on the history of the concept of neuroglia. Glia 1988;1(1):2-9.

[3] Magistretti PJ, Allaman I. A cellular perspective on brain energy metabolism and functional imaging. Neuron 2015;86(4):883-901.

[4] Berthet C, Lei H, Thevenet J, Gruetter R, Magistretti PJ, Hirt L. Neuroprotective role of lactate after cerebral ischemia. J Cereb Blood Flow Metab 2009;29(11):1780-9.

[5] Sada N, Lee S, Katsu T, Otsuki T, Inoue T. Targeting LDH enzymes with a stiripentol analog to treat epilepsy. Science 2015;347(6228):1362-7. 
[6] Pellerin L, Magistretti PJ. Glutamate uptake into astrocytes stimulates aerobic glycolysis: a mechanism coupling neuronal activity to glucose utilization. Proc Natl Acad Sci 1994:91(22):10625-9.

[7] Phelps $\mathrm{CH}$. Barbiturate-induced glycogen accumulation in brain. an electron microscopic study. Brain Res 1972;39(1):225-34.

[8] Marchand I, Chorneyko K, Tarnopolsky M, Hamilton S, Shearer J, Potvin J, Graham TE. Quantification of subcellular glycogen in resting human muscle: granule size, number, and location. J Appl Physiol 2002;93(5):1598-607.

[9] Allaman I. Glial glycogen metabolism. Technical report. Academic Press; 2009.

10] Titze B, Genoud C. Volume scanning electron microscopy for imaging biological ultrastructure. Biol Cell 2016.

[11] Calì C, Baghabra J, Boges DJ, Holst GR, Kreshuk A, Hamprecht FA, Srinivasan M, Lehväslaiho $\mathrm{H}$, Magistretti PJ. 3D immersive virtual reality for studying cellular compartments in 3D models from EM preparations of neural tissues. J Comput Neurol 2016;524(1):23-38.

[12] Vogelstein J.T., Amunts K., Andreou A., Angelaki D., Ascoli G., Bargmann C., Burns R., Cali C., Chance F., Chun M., et al. Grand challenges for global brain sciences. arXiv preprint:1608065482016.

[13] Jorstad A, Nigro B, Cali C, Wawrzyniak M, Fua P, Knott G. Neuromorph: a toolset for the morphometric analysis and visualization of 3D models derived from electron microscopy image stacks. Neuroinformatics 2015;13(1):83-92.

[14] Rajendiran N, Durrant JD. Pyrite: a blender plugin for visualizing molecular dynamics simulations using industry-standard rendering techniques. J Comput Chem 2017.

[15] Asadulina A, Conzelmann M, Williams EA, Panzera A, Jékely G. Object-based representation and analysis of light and electron microscopic volume data using blender. BMC Bioinform 2015;16(1):229.

[16] Beyer J, Al-Awami A, Kasthuri N, Lichtman JW, Pfister H, Hadwiger M. ConnectomeExplorer: query-guided visual analysis of large volumetric neuroscience data. IEEE Trans Vis Comput Graph 2013;19(12):2868-77.

17] Mohammed H, Al-Awami AK, Beyer J, Cali C, Magistretti P, Pfister H, Hadwiger M. Abstractocyte: a visual tool for exploring nanoscale astroglial cells. IEEE Trans Vis Comput Graph 2018;24(1):853-61. (Proceedings IEEE Scientific Visualization 2017)

[18] Vogelstein JT, Mensh B, Häusser M, Spruston N, Evans AC, Kording K, Amunts K, Ebell C, Muller J, Telefont M, et al. To the cloud! a grassroots proposal to accelerate brain science discovery. Neuron 2016;92(3):622-7.

[19] Al-Awami AK, Beyer J, Strobelt H, Kasthuri N, Lichtman JW, Pfister H, Hadwiger M. Neurolines: a subway map metaphor for visualizing nanoscale neuronal connectivity. IEEE Trans Vis Comput Graph 2014;20(12):2369-78.

20] Mächler P, Wyss MT, Elsayed M, Stobart J, Gutierrez R, von Faber-Castell A, Kaelin V, Zuend M, San Martín A, Romero-Gómez I, et al. In vivo evidence for a lactate gradient from astrocytes to neurons. Cell Metab 2016;23(1):94-102.

21] Calì C, Agus M, Gagnon N, Hadwiger M, Magistretti PJ. Visual analysis of glycogen derived lactate absorption in dense and sparse surface reconstructions of rodent brain structures. In: Smart tools and applications for graphics. Eurographics Italian Chapter; 2017. p. 1-8.

22] Wu Y, Whiteus C, Xu CS, Hayworth KJ, Weinberg RJ, Hess HF, De Camilli P. Contacts between the endoplasmic reticulum and other membranes in neurons. Proc Natl Acad Sci 2017;114(24):E4859-67.

[23] Calì C, Marchaland J, Spagnuolo P, Gremion J, Bezzi P. Chapter 20: regulated exocytosis from astrocytes. Int Rev Neurobiol 2009;85:261-93.

[24] Bélanger M, Allaman I, Magistretti PJ. Brain energy metabolism: focus on astrocyte-neuron metabolic cooperation. Cell Metab 2011;14(6):724-38.

[25] Suzuki A, Stern SA, Bozdagi O, Huntley GW, Walker RH, Magistretti PJ, Alberini CM. Astrocyte-neuron lactate transport is required for long-term memory formation. Cell 2011;144(5):810-23.

[26] Oe Y, Baba O, Ashida H, Nakamura KC, Hirase H. Glycogen distribution in the microwave-fixed mouse brain reveals heterogeneous astrocytic patterns. Glia 2016;64(9):1532-45.

[27] Knott G, Marchman H, Wall D, Lich B. Serial section scanning electron microscopy of adult brain tissue using focused ion beam milling. J Neurosci 2008;28(12):2959-64.

[28] Thévenaz P, Ruttimann UE, Unser M. A pyramid approach to subpixel registration based on intensity. IEEE Trans Image Process 1998;7(1):27-41.

[29] Borrett S, Hughes L. Reporting methods for processing and analysis of data from serial block face scanning electron microscopy. J Microsc 2016.

[30] Kaynig V, Vazquez-Reina A, Knowles-Barley S, Roberts M, Jones TR, Kasthuri N, Miller E, Lichtman J, Pfister $\mathrm{H}$. Large-scale automatic reconstruction of neuronal processes from electron microscopy images. Med Image Anal 2015;22(1):77-88.

[31] Berning M, Boergens KM, Helmstaedter M. SegEM: efficient image analysis for high-resolution connectomics. Neuron 2015;87(6):1193-206.

[32] Haehn D, Kaynig V, Tompkin J, Lichtman JW, Pfister H. Guided proofreading of automatic segmentations for connectomics. CoRR 2017. abs/1704.00848

[33] Cardona A, Saalfeld S, Schindelin J, Arganda-Carreras I, Preibisch S, Longair M, Tomancak P, Hartenstein V, Douglas RJ. TrakEM2 software for neural circuit reconstruction. PloS one 2012;7(6):e38011.

[34] Schneider-Mizell CM, Gerhard S, Longair M, Kazimiers T, Li F, Zwart MF, Champion A, Midgley FM, Fetter RD, Saalfeld S, et al. Quantitative neuroanatomy for connectomics in drosophila. Elife 2016;5:e12059.

[35] Sommer C, Straehle C, Köthe U, Hamprecht FA. Ilastik: interactive learning and segmentation toolkit. In: Proceedings of the 2011 IEEE international symposium on biomedical imaging: From nano to macro. IEEE; 2011. p. 230-3.

36] Pfister H, Kaynig V, Botha CP, Bruckner S, Dercksen VJ, Hege H-C, Roerdink JBTM. Visualization in connectomics. CoRR 2012. abs/1206.1428
[37] Lau C, Ng L, Thompson C, Pathak S, Kuan L, Jones A, Hawrylycz M. Exploration and visualization of gene expression with neuroanatomy in the adult mouse brain. BMC Bioinform 2008;9(1):153.

[38] Xia M, Wang J, He Y. BrainNet viewer: a network visualization tool for human brain connectomics. PLOS One 2013;8(7):1-15. doi:10.1371/journal.pone. 0068910.

[39] Gao JS, Huth AG, Lescroart MD, Gallant JL. Pycortex: an interactive surface visualizer for fMRI. Front Neuroinform 2015;9:23.

[40] Goddard TD, Huang CC, Ferrin TE. Visualizing density maps with UCSF chimera. J Struct Biol 2007;157(1):281-7. Software tools for macromolecular microscopy

[41] Agus M, Marton F, Bettio F, Hadwiger M, Gobbetti E. Data-driven analysis of virtual 3D exploration of a large sculpture collection in real-world museum exhibitions. ACM J Comput Cult Herit 2017;11(1):2:1-2:20.

[42] Jensen HW, Christensen NJ. Photon maps in bidirectional Monte Carlo ray tracing of complex objects. Comput Graph 1995;19(2):215-24.

[43] Jensen HW, Christensen NJ. Efficiently rendering shadows using the photon map. In: Proceedings of the 1995 compugraphics; 1995. p. 285-91.

[44] Jensen HW. Global illumination using photon maps. In: Proceedings of the 1996 eurographics workshop on rendering techniques. London, UK: SpringerVerlag; 1996. p. 21-30. ISBN 3-211-82883-4.

[45] Peter I, Pietrek G. Importance driven construction of photon maps. In: Rendering techniques. Springer; 1998. p. 269-80.

[46] Keiriz J.J.G., Zhan L., Chukhman M., Ajilore O., Leow A.D., Forbes A.G. Exploring the human connectome topology in group studies. arXiv preprint:1706102972017.

[47] Rojas GM, Gálvez M, Vega Potler N, Craddock RC, Margulies DS, Castellanos FX Milham MP. Stereoscopic three-dimensional visualization applied to multimodal brain images: clinical applications and a functional connectivity atlas. Front Neurosci 2014;8:328.

[48] Agus M, Gobbetti E, Iglesias Guitián JA, Marton F. Evaluating layout discrimination capabilities of continuous and discrete automultiscopic displays. In: Proceedings of the fourth international symposium on 3D data processing, visualization and transmission; 2010.

[49] Hänel C, Pieperhoff P, Hentschel B, Amunts K, Kuhlen T. Interactive 3D visualization of structural changes in the brain of a person with corticobasal syndrome. Front Neuroinform 2014;8:42.

[50] Laha B, Sensharma K, Schiffbauer JD, Bowman DA. Effects of immersion on visual analysis of volume data. IEEE Trans Vis Comput Graph 2012;18(4):597606.

[51] Agus M, Gobbetti E, Iglesias Guitián JA, Marton F, Pintore G. GPU accelerated direct volume rendering on an interactive light field display. Comput Graph Forum 2008;27(3):231-40. Proc. Eurographics 2008

[52] Laha B, Bowman DA, Socha JJ. Effects of VR system fidelity on analyzing isosurface visualization of volume datasets. IEEE Trans Vis Comput Graph 2014;20(4):513-22.

[53] Forsberg A, Katzourin M, Wharton K, Slater M, et al. A comparative study of desktop, fishtank, and CAVE systems for the exploration of volume rendered confocal data sets. IEEE Trans Vis Comput Graph 2008;14(3): 551-563.

[54] Alper B, Hollerer T, Kuchera-Morin J, Forbes A. Stereoscopic highlighting: 2D graph visualization on stereo displays. IEEE Trans Vis Comput Graph 2011;17(12):2325-33.

[55] Ware C, Mitchell P. Visualizing graphs in three dimensions. ACM Trans Appl Percept (TAP) 2008;5(1):2.

[56] Brown AM, Ransom BR. Astrocyte glycogen and brain energy metabolism. Glia 2007;55(12):1263-71.

[57] Barros LF. Metabolic signaling by lactate in the brain. Trends Neurosci 2013;36(7):396-404.

[58] Commoner B, Lipkin D. The application of the Beer-Lambert law to optically anisotropic systems. Science 1949;110(2845).

[59] Jensen HW. Realistic image synthesis using photon mapping, 364. Ak Peters Natick; 2001.

[60] Derouiche A, Haseleu J, Korf H-W. Fine astrocyte processes contain very small mitochondria: glial oxidative capability may fuel transmitter metabolism. Neurochem Res 2015;40:2402-13.

[61] Harrower M, Brewer CA. Colorbrewer.org: an online tool for selecting colour schemes for maps. Cartograph J 2003;40(1):27-37.

[62] Kumar KM, Reddy ARM. A fast DBSCAN clustering algorithm by accelerating neighbor searching using groups method. Pattern Recognit 2016;58:39-48.

[63] Cruz-Neira C, Sandin DJ, DeFanti TA. Surround-screen projection-based virtual reality: the design and implementation of the CAVE. In: Proceedings of the twentieth annual conference on computer graphics and interactive techniques. ACM; 1993. p. 135-42.

[64] Hasan MS, Yu H. Innovative developments in $\mathrm{HCI}$ and future trends. Int J Autom Comput 2017;14(1):10-20.

[65] Calì C, Agus M, Boges D, Hadwiger M, Magistretti JP. Data from: GLAM: Glycogen-derived lactate absorption modeling for visual analysis of dense and sparse surface reconstructions of rodent brain structures on desktop systems and virtual environments. Dryad Digital Repository; 2018. doi:10.5061/dryad. 808k4r0. VR Data Interact executable file + application data files folder

[66] Cignoni P, Callieri M, Corsini M, Dellepiane M, Ganovelli F, Ranzuglia G. MeshLab: an open-source mesh processing tool. In: Proceedings of the 2008 eurographics Italian chapter conference; 2008. p. 1-8.

[67] Rich L, Brown AM. Glycogen multiple roles in the CNS. Neuroscientist 2016. 1073858416672622 
[68] Lewis TL, Turi GF, Kwon S-K, Losonczy A, Polleux F. Progressive decrease of mitochondrial motility during maturation of cortical axons in vitro and in vivo. Curr Biol 2016;26(19):2602-8.

[69] Sheng Z. The interplay of axonal energy homeostasis and mitochondrial trafficking and anchoring. Trends Cell Biol 2017.

[70] Mania K, Ellis S, Billinghurst M, Steed A. Usability evaluation techniques for virtual reality technologies. In: Proceedings of the 2002 IEEE on virtual reality. IEEE; 2002. 299-299

[71] Brooke J, et al. Sus - a quick and dirty usability scale. Usability Eval Ind 1996;189(194):4-7.
[72] Salvatore L, Christina K. Simple guidelines for testing VR applications. In: Advances in human computer interaction. InTech; 2008. Pellerin L. Distribution of the monocarboxylate transporter MCT2 in human 927 cerebral cortex: an immunohistochemical study. Brain Res 2008;1226:61-9. 928

[74] Hadwiger M, Al-Awami AK, Beyer J, Agus M, Pfister H. SparseLeap: efficient 929 empty space skipping for large-scale volume rendering. IEEE Trans Vis Comput 930 Graph 2018;24(1):974-83. (Proceedings IEEE Scientific Visualization 2017) 